\title{
Principles of Diagnostics and Treatment of Patients with Takayasu Arteritis
}

\author{
Nazirov FG, Suyumov AS, Bahritdinov FSh, Sharapov NU and Khaybullina ZR* \\ Auctioneers society "Republican Specialized Centre of surgery named after academician V. Vakhidov", Uzbekistan
}

Received: 15 September, 2017; Accepted: 02 October, 2017; Published: 20 October, 2017

*Corresponding author: Khaybullina ZR, Auctioneers society "Republican Specialized Centre of surgery named after academician V. Vakhidov", Uzbekistan, E-mail: zrkhaybullina1@gmail.com

\begin{abstract}
Urgency of problem: Many types of vasculitis syndromes are rare and intractable diseases with unknown etiology and pathogenesis; these vasculitis are relatively prevalent and difficult to treat, among such diseases Takayasu Arteritis (TA). Takayasu Arteritis is a nonspecific large vessel vasculitis characterized by granulomatous inflammation of the vessel wall, predominantly with the damage of aorta, aortic arch and its major branches [Toyoshima S, et al. 2008; Keser G, et al. 2014]. In the initial stage of the disease, non-specific constitutional symptoms such as fever, malaise and weight loss may occur. Later, inflammation of the involved arteries progresses, resulting in segmental stenosis, occlusion, dilatation and/ or aneurysm. TA can show different patterns of arterial involvement, disease expression and prognosis in different regions of the world [Brunner J, et al. 2010]. So, there are no universal clinical recommendations and protocols of the TA managing.

The purpose of our work was to evaluate results of medical and surgical treatment of TA in Uzbekistan (Asia) and offer algorithms of diagnostics, clinical management and treatment of TA. There were analyzed 210 patients with TA, young female patients were predominant (male $/$ female ratio $=$ 1:7.3; middle age $=32.1 \pm 1.3$ years); brachio-cephal arteries damage $(100 \%)$. Combined lesion (aortic arch + thoracic or abdominal or renal arteries) was in 58\% of patients. The coronary, renal arteries and pulmonary arteries also be involved in some patients. Many studies use a combination of clinical symptoms sometimes linked to changes in acute-phase reactants. We believe, that Duplex Scan (DS) is very important for detection of stenosis/occlusion of arteries and useful for evaluation of inflammation intensity in the complex with biochemical inflammatory markers such as C-reactive protein, leucocytes-count, RBS sedimentation velocity. To reach the main purpose of the medical treatment of TA- conversion of the acute phase of TA to morphological remission, glucocorticoid treatment is necessary. The typical initial dose is approximately 20 to $30 \mathrm{mg} / \mathrm{day}$ prednisolone (PSL), though the dose should be adjusted according to the age and constitution of the patient. Our investigation shows that in acute and subacute phase of TA disease prolonged using of glucocorticoid in "small" doses (10-30 mg/day) is less effective. We have received good results from pulse-therapy. Scheme of medical treatment is $1000 \mathrm{mg}$ of cyclophosphamide and $1000 \mathrm{mg}$ PSL intravenous at the first day, then, at the second and third days $-1000 \mathrm{mg}$ PSL per day. After this treatment level of the CRP, RBC sedimentation decreased $(\mathrm{p}<0.05)$ in contrast pretreatment level. After pulse-therapy we used methotrexate. Surgical treatments have been performed after full programme of medical treatment and normalization of inflammation markers and ultrasound signs.
\end{abstract}

Conclusion: The most informative imaging methods of TA diagnostics are DS, RA and MSCT-angiography. On the base of the DS, MSCT-angiography and laboratory tests data (CRP, RBC sedimentation velocity, leucocytes count), medical and surgical treatment strategy can be developed. Medical treatment bu pulse-therapy with prednisolone and cyclophosphamide is the most effective in contrast medical treatment with small doses of glucocorticoids. Surgical treatment should be performed after clinical and laboratory remission (not less then 3 months), in the chronically stage of the disease. In the cases, when artery for implantation was intact and not damaged, extra-thoracic reconstructive surgery should be preferred.

\section{Introduction}

Many types of vasculitis syndromes are rare and intractable diseases with unknown etiology and pathogenesis; these vasculitis are relatively prevalent and difficult to treat, among such diseases Takayasu Arteritis (TA) [18]. Takayasu arteritis is a nonspecific large vessel vasculitis characterized by granulomatous inflammation of the vessel wall, predominantly with the damage of aorta, aortic arch and its major branches [19]. Takayasu arteritis is classified into four types on the basis of anatomy of the affected arteries: (I) Arteritis affecting the aortic arch and its major branches; (II) Arteritis affecting the thoracic/abdominal aorta (III); Arteritis affecting the entire aorta (IV); Arteritis affecting the pulmonary artery [11]. In 1997,
Numano, et al. proposed a new method of classification based on angiographic findings into 5 types with sub categorization by the presence/absence of lesions in the coronary and pulmonary arteries: type I: branches of the aortic arch; type IIa: ascending aorta, aortic arch and its branches; type IIb: IIa lesions + thoracic descending aorta; type III:scending aorta, abdominal aorta, and renal arteries; type IV:abdominal aorta and/or renal arteries;type V: type IIb+IV(ascending aorta, aortic archand/or renal arteries) $[13,21]$.

There are classification scheme, which is provided for aortitis and periaortitis based on the pattern of the inflammatory infiltrate: granulomatous/giant cell pattern, lymphoplasmacytic 
pattern, mixed inflammatory pattern, and the suppurative pattern. These inflammatory patterns are discussed in relation to specific systemic diseases including giant cell arteritis [19].

TA predominantly affects young females during the second or third decades of life and mainly involves the aortic arch and its primary branches, ascending aorta, thoracic descending aorta and abdominal aorta $[9,22]$. In the initial stage of the disease, non-specific constitutional symptoms such as fever, malaise and weight loss may occur [23]. Later, inflammation of the involved arteries progresses, resulting in segmental stenosis, occlusion, dilatation and/or aneurysm [12]. This may cause extremity pain, claudication, bruits, absent or diminished pulses and loss of blood pressure. TA generally follows an insidious course, however, presentation with acute visual loss or stroke may also occur [22]

Arnaud, et al. generally consider the disease as being active in the presence of the following criteria: firstly, the presence of constitutional or ischemic symptoms; secondly, the increased acute phase reactants; thirdly, the mural contrast enhancement in CT-scan or fourthly, the mural contrast enhancement or signal abnormalities in MRI; fifthly, the abnormal vascular uptake in PET-scan. When TA is active, our follow-up recommendation is to perform an ultrasonography of the supra-aortic vessels and an aortic MRI or CT-scan twice a year. When TA appears to be inactive, we recommend to perform these investigations once a year. Surgical treatment of TA is limited to a few indications. The overall prognosis of TA is good but the quality of life is altered. Management of TA patients is difficult because of the lack of reliable diagnostic criteria, consensual therapeutic strategies and validated disease activity criteria [24].

TA can show different patterns of arterial involvement, disease expression and prognosis in different regions of the world $[6,8]$. In Japan, where this disease was first opened (the name "Takayasu disease" was first used by Yasuzo Niimi in 1942), TA is included in the Disease List for the Specific Disease Treatment Research Program which provides Medical Care Certificates to patients and reimburses a portion of healthcare expenses [6]

So, there are no universal clinical recommendations and protocols of the TA managing.

The purpose of our work was to evaluate results of medical and surgical treatment of TA in Uzbekistan (Asia) and offer algorithms of diagnostics, clinical management and treatment of TA.

\section{Material and Methods}

There were analyzed 210 patients with TA. Diagnostically procedures included common clinical examination, laboratory tests, neurological status evaluation, as well as Duplex Scan (DS) by device Logiq P6, GE Healthcare; X-ray-contrast angiography on device PHILIPS Integris HM-3000; multi-spiral computer tomography(MSCT) on device General Electric Optima. In some cases MSCT angiography of the aorta and its major branches was done after reconstruction of the aortic arch and/ or after reconstruction of the renal arteries. Marker of systemic inflammation-C-reactive protein (CRP) have been detected on the full automatic biochemical analyzer VITROS-350; Red Blood Cells (RBC) sedimentation velocity detected by Westergren method, leukocyte count detected by hematological analyzer BC 5800 Mindray. Statistical method was ANOVA.

\section{Results}

There were examined 210 patients with TA, young female patients were predominant (male/female ratio $=1: 7.3$; middle age $=32.1 \pm 1.3$ years); brachio-cephal arteries damage $(100 \%)$. Combined lesion (aortic arch + thoracic or abdominal or renal arteries) was in $58 \%$ of patients. The coronary, renal arteries, and pulmonary arteries also be involved in some patients. Clinical manifestations range was from asymptomatic disease with impalpable pulses to subclavian steal syndrome to catastrophic neurologic strokes. It has been established that typical clinical signs and symptoms of TA were caused by cerebral ischemia (dizziness, headache), by inflammation (fever, malaise, cervical lymphadenopathy, easy fatigability), by stenosis of affected vessel (abnormal pulse and blood pressure, heart murmurs, vascular bruits in the neck, back and abdomen).

The most informative imaging methods of aorta and its arch with major branches' stenosis are DS, RA and MSCT-angiography [2].

Despite many attempts to adopt standardized approaches to disease activity assessment in Large Vessel Vasculitis (LVV), no one measure or set of measures has been accepted as valid and useful for clinical trials [1]. Many studies use a combination of clinical symptoms sometimes linked to changes in acutephase reactants. In terms of a single activity measure, the Birmingham Vasculitis Activity Score (BVAS) is an index that has been developed and best validated for use in Antineutrophil Cytoplasmic Antibody (ANCA) -associated vasculitis, but few studies of LVV have incorporated BVAS [10].

We believe, that DS is very important for detection of stenosis/ occlusion of arteries and useful for evaluation of inflammation intensity in the complex with biochemical inflammatory markers [20]. Ultrasonic findings of TA is a strong thickness of the wall of aorta and it's major branches, thickness of common carotid arteries walls was even, prolonged and circular; walls of the thoracic and abdominal aorta were very thick, that caused its stenotic lesion and narrowing. Degree of thickening depended on intensity of the inflammation intensity [3]. Acute and active inflammation characterizes by washed away sidebars of the vessel's wall on the ultrasound picture. Decreasing of inflammation after medical treatment associated with decreasing of thickening of aortas wall and walls of aortic branches. Ultrasound signs of calcification of aorta and its major branches indicate prolonged damage of the vessels. So, DS is a reliable objective method of the estimation to activity of TA.

Differential diagnostics of TA can include three broad categories of inflammatory aortic disease, which in order of increasing inflammation, they are atherosclerosis, atherosclerosis with excessive inflammation, and periaortitis [19]. Atherosclerosis is most often associated with at least some degree of inflammation, and in some cases, the inflammation can be prominent [16]. Most of the vasculitis may co morbidities such as breast diseases, abdominal inflammatory diseases [25, 26].

MSCT- angiography is useful in the diagnosis of TA, method 
is non invasive and have a lot of advantages such as high quality of imaging, easy to perform, small time for procedure and possibility to visualizations not only bright spot, but also walls of the aorta and surrounding soft tissues, possibility of the building 3D-reconstruction, significant reduction of the X-ray dose in contrast with traditional angiography [7]. On the base of the DS, MSCT-angiography and laboratory tests data, medical and surgical treatment strategy can be developed.

As well known, the main purpose of the medical treatment is conversion of the acute phase of TA to morphological remission. Glucocorticoid treatment is the gold standard in the medical treatment of Takayasu arteritis [9]. The results of treatment trials with conventional immunosuppressive agents such as methotrexate, azathioprine, mycophenolate mofetil, and cyclophosphamide have overall been disappointing. TNF- $\alpha$ blockers are ineffective in giant cell Arteritis, while observational evidence and a phase 2 randomized trials support the use of tocilizumab in relapsing giant cell arteritis. Observational evidence strongly supports the use of anti-TNF- $\alpha$ agents and tocilizumab in TA patients with relapsing disease [15].

Patients generally respond well to glucocorticoid. The typical initial dose is approximately 20 to $30 \mathrm{mg} /$ day prednisolone (PSL), though the dose should be adjusted according to the age and constitution of the patient [11]. Our investigations shows that in acute and subacute phase of TA disease prolonged using of glucocorticoid in "small" doses (10-30 mg/day) is less effective. We have received good results from pulse-therapy. Scheme of medical treatment is $1000 \mathrm{mg}$ of cyclophosphamide and 1000 mg PSL intravenous at the first day, then, at the second and third days-1000 mg PSL per day. After this treatment level of the CRP, RBC sedimentation decreased $(p<0.05)$ in contrast pretreatment level. After pulse-therapy we used methotrexate.

Surgical treatment have been performed after full course of medical treatment and normalization of inflammation markers and ultrasound signs. These is correlate with JCS recommendations, when surgical treatment of occlusions and dilatations due to TA should in principle be performed during periods in which patients do not exhibit severe inflammation and are not on glucocorticoid therapy $[11,12]$. We performed surgical treatment in the chronically stage of the TA disease. We used different types of reconstructive operations on the aortic arch and its major branches, such as intra-thoracic and extra-thoracic surgical treatment [4].

As well known, bypass surgery is the standard technique. According guidelines, surgery should be performed when inflammation is not present and the grafts should be anastomosed to intact vessels; branches of the aortic arch are bypassed with artificial grafts, while the renal arteries are bypassed with artificial grafts or venous grafts [14,17].

According our data surgical treatment should be performed 3-4 months after beginning of medical treatment. In the cases, when artery for implantation was intact and not damaged, extra-thoracic reconstructive surgery was preferred. When that artery was absent, intra-thoracic bypass surgery was performed. According our experience, extra-thoracic surgery was predominant in the most of cases $[3,4,5]$. Surgical treatment is indicated when stenosis of brachial-cephalic arteries is $70 \%$ or more; in the case of occlusion of truncus brachiocephalicus and common carotid arteries; bypass surgery is preferred.

Syndrome of vertebral-subclavicular deficits and subcompensated or de-compensated ischemia of upper limbs are indications to surgery in proximal portion of subclavicular arteries [5,20].

\section{Conclusion}

The most informative imaging methods of TA diagnostics are DS, RA and MSCT-angiography. On the base of the DS, MSCTangiography and laboratory tests data (CRP, RBC sedimentation velocity, leucocytes count), medical and surgical treatment strategy can be developed. Medical treatment by pulse-therapy with prednisolon and cyclophosphamid is the most effective in contrast medical treatment with small doses of glucocorticoids. Surgical treatment should be performed after clinical and laboratory remission (not less then 3 months), in the chronically stage of the disease. In the cases, when artery for implantation was intact and not damaged, extra-thoracic reconstructive surgery should be preferred.

\section{References}

1. Aydin Sibel Zehra, Haner Direskeneli, Antoine Sreih, Fatma Alibaz-Oner, Ahmet Gul, Sevil Kamali, et al. Update on Outcome Measure Development for Large Vessel Vasculitis: Report from OMERACT 12. J Rheumatol. 2015;42(12):2465-2469. doi: 10.3899/ jrheum.141144

2. Bakhritdinov FSh, Suyumov AS. Multispiral computer tomography in diagnosis of vascular pathology in patients with nonspecific aortoarteritis. Journal of Angiology and vascular surgery. 2015;21(4):63.

3. Bakhritdinov FSh, Suyumov AS. Surgical treatment of a female patient with non-specific aortoarteritis. Journal of Angiology and vascular surgery. 2015;21(2):183-191.

4. Bakhritdinov FSh, Suyumov AS. Surgical treatment of a female patient with type III nonspecific aortoarteritis. Journal of Angiology and vascular surgery (in Russian, Moscow). 2016;22(2):145-151.

5. Bakhritdinov FSh, Suyumov AS, Sharapov NU. Outcomes of surgical management of patients with nonspecific aortoarteriitis with isolated lesion of brachio-cephalic arteries. Journal of Angiology and vascular surgery. 2016;22(4):144-150.

6. Brunner J, Feldman BM, Tyrrell PN, Kuemmerle-Deschner JB, Zimmerhackl LB, Gassner I, et al. Takayasu arteritis in children and adolescents. Rheumatology. 2010;49(10):1806-1814. doi: 10.1093/ rheumatology/keq167

7. Bicakcigil M, Aksu K, Kamali S, Ozbalkan Z, Ates A, Karadag O, et al. Takayasu's arteritis in Turkey - clinical and angiographic features of 248 patients. Clin Exp Rheumatol. 2009;27(Suppl 52):S59-64.

8. Clinical Features of Takayasu Arteritis MHLW in 1998).

9. Keser G, Direskeneli H, Aksu K. Management of Takayasu arteritis: a systematic review. Rheumatology (Oxford). 2014;53(5):793-801. doi: 10.1093/rheumatology/ket320

10. Henes JC, Mueller M, Pfannenberg C, Kanz L, Koetter I. Cyclophosphamide for large vessel vasculitis: assessment of response by PET/CT. Clin Exp Rheumatol. 2011;29(1 Suppl 64):S4348. 
11. JCS Guidelines for Management of Vasculitis Syndrome. Circulation Journal. 2011;75.

12. Kerr GS, Hallahan CW, Giordano J, Leavitt RY, Fauci AS, Rottem M, et al. Takayasu arteritis. Ann Intern Med. 1994;120(11):919-929.

13. Kobayashi Y, Numano F. Takayasu arteritis. sakura Publishing. 2001;192-198.

14. Liang P, Hoffman GS. Advances in the medical and surgical treatment of Takayasu arteritis. Curr Opin Rheumatol. 2005;17(1):16-24.

15. Muratore F, Pipitone N, Salvarani C. Standard and biological treatment in large vessel vasculitis: guidelines and current approaches. Expert Rev Clin Immunol. 2017;13(4):345-360. doi: 10.1080/1744666X.2017.1285699

16. Nazirov FG, Khaybullina ZR, Kosnikova IV, Kim VH. Inflammation and reactive oxygen species generation at the peripheral and coronary atherosclerosis, comparative evaluation of its intensity and grade. J Cardiovascular Thoracic Surgery 2016;1(1): 5.

17. Parra JR, Perler BA. Takayasu's disease. Semin Vasc Surg. 2003;16(3):200-208.

18. Pokrovsky AV, Zotikov AE, Yudin VI. Nonspecific aortoarteriitis (Takayasu's disease). Moscow: IRSIS, 2002.

19. Stone JR, Bruneval P, Angelini A, Bartoloni G, Basso C, Batoroeva $\mathrm{L}$, et al. Consensus statement on surgical pathology of the aorta from the Society for Cardiovascular Pathology and the Association for European Cardiovascular Pathology: I. Inflammatory diseases. Cardiovasc Pathol. 2015;24(5):267-278. doi: 10.1016/j. carpath.2015.05.001
20. Suyumov AS. Clinical case report of a female patient with type IV takayasu arteritis. Journal of Angiology and vascular surgery. 2016;22(3):157-162.

21. Toyoshima S, Tasaka K, Ozaki S. Immunology for Medicine and Pharmacology. Second Edition. 2008;163.

22. Watts R, Al-Taiar A, Mooney J, Scott D, Macgregor A. The epidemiology of Takayasu arteritis in the UK. Rheumatology (Oxford). 2009;48(8):1008-1011. doi: 10.1093/rheumatology/ kep153

23. Wipfler-Freissmuth E, Moosig F, Schirmer M. Recommendations of the European League Against Rheumatism (EULAR) for the treatment of "large-vessel vasculitides. Z Rheumatol. 2009;68(3):260, 262263. doi: $10.1007 / \mathrm{s} 00393-008-0387-1$

24. Arnaud L, Haroche J, Piette JC, Amoura Z. Takayasu arteritis: a French single centre experience. Rev Med Interne. 2010;31(3):208215. doi: 10.1016/j.revmed.2009.01.011

25. Arda Isik, Deniz Firat. Bilateral intra-areolar polythelia. Breast J. 2017. doi: $10.1111 /$ tbj.12838

26. Isik A, Idiz O, Firat D. Novel Approaches in Pilonidal Sinus Treatment. Prague Med Rep. 2016;117(4):145-152. doi: 10.14712/23362936.2016.15 